\title{
DERMATOGLIFIA E COMPOSIÇÃO CORPORAL EM APNÉIA OBSTRUTIVA DO SONO
}

\author{
Luiz Bittencourt Mercanti ${ }^{1}$, Marcio L. de S. Bezerra ${ }^{2}$, \\ José Fernandes Filho ${ }^{3}$, Claudio José Struchiner ${ }^{4}$
}

\begin{abstract}
RESUMO - A síndrome da apnéia obstrutiva do sono (SAOS) tem como principal fator de risco a obesidade e acredita-se que fatores genéticos poderiam contribuir na sua patogênese. O objetivo deste estudo foi descrever as características antropométricas e dermatoglíficas dos portadores de SAOS. Foram mensurados: indice de massa corporal (IMC); indice de conicidade, gordura corporal relativa, somatotipo e coletadas as impressões digitais. Trinta e um casos de SAOS foram comparados a número igual de controles. Pelo IMC e gordura corporal relativa os apnéicos foram classificados como obesos. O indice de conicidade revelou forte componente de obesidade central. No somatotipo, predominou a categoria endomorfo-mesomorfo, indicando alta magnitude dos componentes músculo-esquelético e adiposo com linearidade relativa de grande volume por unidade de altura. Para índices mais graves de apnéia observa-se maior predominância mesomórfica na composição corporal. Através do teste $t$, a dermatoglifia não mostrou diferenças estatisticamente significativas entre portadores da SAOS e os controles.
\end{abstract}

PALAVRAS-CHAVE: apnéia obstrutiva do sono, composição corporal, dermatoglifia.

\begin{abstract}
Dermatoglyphics and body composition in obstructive sleep apnea
ABSTRACT - Obesity is the main risk factor for obstructive sleep apnea syndrome (OSAS) and genetic patterns can modulate the pathogenesis of the disease. The aim of this study is to describe the anthropometrics and dermatoglyphics features among OSAS carriers. We collected information on Body Mass Index (BMI), Conicity Index (CI), Body Fat Mass (BFM), somatotype and fingerprints. Thirty-one cases of OSAS were compared to an equal number of controls. Membership to the obese category is based on observed BMI and BFM. The $\mathrm{Cl}$ distribution among cases shows a strong central obesity component. The endomorph-mesomorph somatotype category predominates among cases showing high adiposity and relative muscle-skeletic development, such as relative linearity of great mass per unit of height. Increased morbidity, as given by more serious indices of apnea, correlates positively with higher mesomorphic predominance in the body composition. Analysis of dermatoglyphic data does not show significant statistical differences between OSAS patients and controls.
\end{abstract}

KEY WORDS: obstructive sleep apnea syndrome, body composition, dermatoglyphics.

A síndrome da apnéia obstrutiva do sono (SAOS) caracteriza-se pela obstrução completa ou parcial da via aérea superior, na região orofaríngea, devido ao relaxamento da língua e dos tecidos moles durante o sono'. A má qualidade do mesmo resulta, entre outros sintomas, em excessiva sonolência diurna. Porém, nem sempre a sonolência diurna é causada pela SAOS. Há fatores anatômicos e fisiológicos, que isoladamente ou associados, contribuem na sua etiologia, ora podendo advir da narcolepsia ${ }^{2}$ ou da distrofia miotonica ${ }^{3}$, esta última causando predominantemente a apnéia do sono cen- tral. Pode ainda advir de mal formação congênita observada na Seqüência de Pierre Robin ${ }^{4}$.

Estudos prévios de famílias isoladas têm sugerido que fatores hereditários podem ser importantes na patogênese da SAOS. O papel da hereditariedade foi avaliado pelo grau de agrupamento familiar dos sintomas associados à síndrome, ou seja, pela coincidência entre parentes com o(s) mesmo(s) sinto$\mathrm{ma}(\mathrm{s})^{5}$.

Por outro lado, há estudos que sugerem predisposição genética na transmissão de algumas doenças, a partir da análise das características dermato-

\footnotetext{
Universidade Castelo Branco, Rio de Janeiro RJ, Brasil (UCB): 'Mestrado em Ciência da Motricidade Humana - UCB, Avaliador Funcional - Physigraph - RJ; ${ }^{2}$ Doutorando em Neurologia - Universidade Federal Fluminense, Niterói RJ, Brasil (UFF) Coordenador Técnico do Instituto de Estudos do Sono - UNESA - RJ; ${ }^{3}$ Doutorado em Educação Física - VNIIFK, Moscou, Rússia, Professor Titular do Mestrado em Ciência da Motricidade Humana - UCB; ${ }^{4}$ Doutorado em Epidemiologia - Harvard University, Harvard, Estados Unidos, Pesquisador Titular da Fundação Oswaldo Cruz - FIOCRUZ, Pesquisador CNPq - FAPERJ.
}

Recebido 9 Outubro 2003, recebido na forma final 25 Março 2004. Aceito 11 Maio 2004.

Dr. Luiz Bittencourt Mercanti - Rua Décio Vilares 203/402 - 222041-040 Rio de Janeiro RJ - Brasil. 
glíficas dos indivíduos, isto é, de acordo com a similaridade das impressões digitais, como por exemplo, diabetes mellitus tipo $I^{6}$. Sabe-se que os dermatóglifos são sistemas de cristas dermopapilares formados durante o terceiro ou quarto mês de vida intra-uterina e compõem arranjos característicos praticamente imutáveis, constituindo-se em marca genética, pois uma vez formados, mudam apenas de tamanho com o crescimento. Por esta razão, há vários anos os estudos dermatoglíficos são aplicados enfaticamente na Clínica Médica, pelo fato de uma série de anomalias congênitas determinadas por aberrações cromossômicas estarem associadas a características dermatoglíficas incomuns ${ }^{7}$.

Assim o presente estudo tem por objetivo descrever os perfis antropométrico e dermatoglífico dos portadores da SAOS, a fim de agregar dados potencialmente valiosos aos sinais e sintomas sugestivos para a síndrome, auxiliando os médicos na seleção e encaminhamento dos indivíduos para a polissonografia.

\section{MÉTODO}

Esta pesquisa utiliza o modelo epidemiológico observacional, do tipo caso-controle ${ }^{8}$. O estudo foi aprovado pela Comissão de Ética da Universidade Castelo Branco e a coleta de dados foi feita, por conveniência, no Instituto de Estudos do Sono da Universidade Estácio de Sá, no Rio de Janeiro. Fizeram parte do estudo 31 indivíduos portadores da SAOS, segundo resultado dos exames de polissonografia, independentemente do nível de gravidade. Para o grupo controle foram selecionados, em número equivalente ao de casos, indivíduos que não apresentassem quaisquer sinais ou sintomas sugestivos de SAOS. Todos os sujeitos, casos e controles, assinaram termo de consentimento autorizando a utilização de seus dados na pesquisa de acordo com a ética médica.

Para se chegar a esta amostra $(n=31)$, foram excluídos anteriormente dois indivíduos cujas impressões digitais impressas no papel não estavam perfeitamente legíveis de forma a permitir análise dermatoglífica adequada, além de vinte e um indivíduos que não realizaram a polissonografia, mesmo apresentando os sintomas sugestivos de SAOS.

\section{Procedimentos}

Utilizamos os seguintes protocolos como procedimento experimental:

- Índice de massa corporal (IMC): determina a relação da massa (peso) corporal para a estatura, permitindo classificar o grau de sobrepeso ou o estado de desnutrição do indivíduo. A correlação entre os valores percentuais de gordura corporal, determinada pela pesagem hidrostática, e o IMC é moderada, sendo $r=0.60$ a $0.80^{9}$; e a correlação entre a densidade corporal determinada pela pesagem hidrostática e o IMC é $r=0.70^{10}$. É calculado pela divisão do peso (massa) corporal pela estatura elevada ao quadrado: IMC = peso $(\mathrm{kg}) /$ estatura $(\mathrm{m})^{2}$.

- Fracionamento da gordura corporal: a adiposidade corporal foi determinada através do método duplamente indireto com a mensuração de três dobras cutâneas que representam o membro superior, tronco e membro inferior. A espessura das dobras cutâneas tem alta correlação com o percentual de gordura corporal, variando r entre 0.70 e $0.90^{11}$. Para tal foram escolhidos protocolos que estimam a densidade corporal a partir do uso de equações generalizadas, para homens $(r=0,89)$ e mulheres $(r=0,84)$ adultos, considerando a idade e o somatório das dobras cutâneas triciptal, supra-ilíaca e anterior da coxa (muIheres); e peitoral, abdominal e anterior da coxa (homens) ${ }^{12,13}$.

- Somatotipo: a técnica do somatotipo é utilizada para estimar a forma corporal e sua composição, resultando num resumo quantitativo da forma física. É expresso por três números que, sempre na mesma ordem, representam os componentes endomorfia (adiposidade); mesomorfia (músculo-esquelética) e ectomorfia (linearidade relativa). Em cada componente, os valores entre 2 e 2,5 são considerados baixos; de 3 a 5, moderados; de 5,5 a 7, altos; e acima de 7, muito altos ${ }^{14}$. Nesta pesquisa, o somatotipo foi calculado pelo método antropométrico ${ }^{15}$. Para calculálo, são necessárias dez medidas: estatura total; peso corporal; quatro dobras cutâneas (tríceps, subescapular, supraespinhal e panturrilha medial); dois diâmetros ósseos (biepicondiliano do úmero e do fêmur); e dois perímetros (braço flexionado em tensão máxima e panturrilha medial).

- Índice de conicidade (IC): pode ter valor clínico quando há a intenção de medir a distribuição da gordura corporal. Neste caso, considera-se o ser humano como um cilindro, no seu extremo mais delgado, e como dois cones com base comum na cintura, para seu extremo mais largo em torno do abdômen. A amplitude teórica para o IC é de 1,00 (cilindro perfeito) a 1,73 (duplo cone perfeito) ${ }^{16}$. A mensuração da cintura se dá no ponto de menor circunferência entre a última costela e a crista ilíaca ${ }^{17}$. A correlação entre o IC e a relação cintura-quadril é de moderada a elevada para a maioria das populações, sendo $r=0,45$ a $0,86^{18}$.

- Dermatoglifia: neste método visa-se obtenção das impressões digitais e seu processamento posterior. Para obtê-las utilizou-se papel branco de densidade e rugosidade médias e almofada de base macia para a impressão $^{19}$. O seu processamento preliminar determina como padrão os tipos de desenho nas falanges distais dos dedos das mãos e a quantidade de linhas formadas pelas 
cristas de pele dentro do desenho. Os tipos de desenho mais comumente encontrados são: arco (ausência de deltas, composto de cristas que atravessam transversalmente a almofada digital); presilha (feixe de linhas paralelas que faz uma volta de 180 graus, possui sempre um delta e classifica-se de acordo com a posição do mesmo na impressão feita no papel, à esquerda - ulnar, à direita -radial) e verticilo (sistema nuclear de linhas formando círculos concêntricos ou espirais possuindo dois deltas, um de cada lado) ${ }^{19}$. A contagem de linhas é feita traçando-se a lápis um segmento de reta (linha de Galton), que une o centro geométrico de cada delta àquele do sistema nuclear. Nesta contagem não se incluem as linhas que constituem os centros do sistema nuclear e do delta. Obviamente, só fazem parte da contagem as presilhas e os verticilos. Nesses últimos faz-se duas contagens, uma para cada delta, sendo o número registrado a média obtida da soma dos dois valores. Caso a soma dos valores indique um número ímpar, acrescenta-se à média o valor de 0,5 para que se obtenha um número inteiro, pois não existe $1 / 2$ linha ${ }^{19}$. Após este procedimento preliminar, são calculados os índices padronizados, fundamentais das impressões digitais: a) a quantidade de cada tipo de desenho entre os 10 dedos das mãos; b) a quantidade de linhas (QL), em cada dedo das mãos; c) a intensidade de padrão (D10), calculado pela soma de deltas de todos os desenhos; d) o somatório da quantidade total de linhas (SQTL) nos 10 dedos das mãos; e) fórmulas digitais que indicam as combinações entre os diferentes tipos de desenho.

\section{Instrumentos}

Foram utilizados os seguintes instrumentos para a coleta dos dados antropométricos e dermatoglíficos:

- Compasso de dobras cutâneas: do tipo calibrador de tensão constante $\left(10 \mathrm{~g} / \mathrm{mm}^{2}\right)$; modelo Lange Skinfold Caliper; fabricado em 1999 por Beta Technology Incorporated, U.S.A.
- Balança antropométrica: mecânica, com estadiômetro acoplado (Martin -1,90m / 0,05m); modelo 31 (150kg $/ 0,1 \mathrm{~kg})$, fabricada em 1998, pelas Industrias Filizola S.A.

- Trena antropométrica: metálica plana em aço carbono, com variações na escala de 0,001m, fabricada em 2001 pela Sanny ${ }^{\circledR}-A M B$.

- Paquímetro: tamanho pequeno, com amplitude de $0 \mathrm{~mm}$ a $350 \mathrm{~mm}$, tipo deslizante em alumínio com variações na escala de 0,001m. Fabricado em 2001 pela Sanny®-AMB.

- Almofada coletora de impressões digitais: tipo de mesa, retangular $(9,0 \mathrm{~cm} \times 6,0 \mathrm{~cm})$, modelo Coletor Impress $\AA$ 350, fabricada em 2001 pela Impress Impressões Digitais Ltda. São Paulo.

\section{Tratamento estatístico}

Estudo descritivo: os dados foram analisados observando-se as medidas de tendência central (média, mediana e moda) e de dispersão da amostra (amplitude, variância, desvio padrão e erro padrão da média).

Estudo analítico: para se analisar a correlação das características antropométricas com o nível de gravidade da SAOS (índice de apnéia / hora) fez-se uso das técnicas de regressão linear (simples e múltipla). Para a comparação entre as características dermatoglíficas dos apnéicos (casos) e dos indivíduos saudáveis (controle) utilizou-se o teste $t$.

Nível de significância: o nível de significância do teste assumido nesta pesquisa foi de $5 \%$ (erro tipo I).

\section{RESULTADOS}

Nesta pesquisa a amostra foi composta por indivíduos portadores da SAOS $(n=31)$, sendo entre eles 9 mulheres e 22 homens, o que corrobora os achados da literatura, que apontam maior incidência da síndrome em homens do que em mulheres ${ }^{20}$.

Tabela 1. Estatística descritiva do índice de apnéia e características antropométricas do grupo de casos $(n=31)$.

\begin{tabular}{lccccc}
\hline & Média & S.E.M. & Desvio Padrão & Amplitude & Variância \\
\hline I A / h & 29,43 & 4,2696 & 23,7723 & 81,80 & 565,124 \\
I C & 1,26 & $1,451 \mathrm{E}-02$ & $8,076 \mathrm{E}-02$ & 0,29 & $6,523 \mathrm{E}-03$ \\
I M C & 30,73 & 0,925 & 5,1504 & 21,56 & 26,527 \\
G C R & 26,30 & 1,0535 & 5,8656 & 22,97 & 34,405 \\
Endomorfia & 6,15 & 0,2273 & 1,2654 & 5,70 & 1,601 \\
Mesomorfia & 6,42 & 0,2883 & 1,6054 & 7,70 & 2,577 \\
Ectomorfia & 0,53 & 0,1437 & 0,8002 & 3,85 & 0,640 \\
\hline
\end{tabular}

IA/h, Índice de apnéia por hora; IC, Índice de conicidade; IMC, Índice de massa corporal; GCR, Gordura corporal relativa (\%); Endomorfia, Constituição adiposa; Mesomorfia, Constituição músculo-esquelética; Ectomorfia, Linearidade relativa. 
Tabela 2. Índice de apnéia e características antropométricas (estratificado por nível de gravidade).

\begin{tabular}{|c|c|c|c|c|c|c|c|c|}
\hline \multirow{2}{*}{$\begin{array}{l}\text { Nível de } \\
\text { Gravidade }\end{array}$} & \multicolumn{7}{|c|}{ Valores médicos por subgrupo } & \multirow[b]{2}{*}{$\mathrm{R} \subset \mathrm{Q}$} \\
\hline & $\mathrm{I} A / \mathrm{h}$ & I M C & IC & G C R & Endo & Meso & Ecto & \\
\hline $5-15(n=11)$ & 9,66 & 28,89 & 1,22 & 23,58 & 5,7 & 5,9 & 0,62 & 0,89 \\
\hline $15-30(n=9)$ & 20,12 & 24,76 & 1,23 & 26,25 & 5,7 & 5,7 & 0,89 & 0,90 \\
\hline$>30(n=11)$ & 56,83 & 34,99 & 1,33 & 29,07 & 6,9 & 7,4 & 0,15 & 0,97 \\
\hline
\end{tabular}

Tabela 3. Soma da quantidade de linhas e tipo de desenho, por dedo, em portadores da síndrome da apnéia obstrutiva do sono $(n=31)$.

\begin{tabular}{lcccccccc}
\hline & Média & S.E.M. & $\begin{array}{c}\text { Desvio } \\
\text { Padrão }\end{array}$ & Amplitude & Variância & \multicolumn{2}{c}{ Predominância (\%) } \\
& & & & & L & A \\
\hline Mesql1 & 14,06 & 1,15 & 6,42 & 25 & 41,26 & 74,2 & 19,4 & 6,4 \\
Mesql2 & 10,42 & 0,99 & 5,49 & 18 & 30,12 & 61,3 & 35,5 & 3,2 \\
Mesql3 & 11,29 & 0,94 & 5,25 & 17 & 27,61 & 67,7 & 22,6 & 9,7 \\
Mesql4 & 14,77 & 0,87 & 4,87 & 22 & 23,71 & 61,3 & 35,5 & 3,2 \\
Mesql5 & 12,61 & 0,66 & 3,67 & 15 & 13,45 & 83,8 & 16,2 & - \\
Mdsql1 & 16,03 & 1,20 & 6,67 & 27 & 44,43 & 58,0 & 35,5 & 6,5 \\
MdsqI2 & 10,77 & 0,92 & 5,12 & 20 & 26,18 & 64,5 & 25,8 & 9,7 \\
Mdsql3 & 11,58 & 0,71 & 3,96 & 17 & 15,65 & 74,2 & 22,6 & 3,2 \\
Mdsql4 & 14,23 & 0,86 & 4,80 & 20 & 23,05 & 45,2 & 54,8 & - \\
MdsqI5 & 12,97 & 0,77 & 4,28 & 18 & 18,30 & 87,1 & 12,9 & - \\
\hline
\end{tabular}

Mesql1 - 5, Mão esquerda, soma da quantidade de linhas da $1^{\text {a }}$ à $5^{\text {a }}$ falange distal; Mdsql1 - 5, Mão direita, soma da quantidade de linhas da $1^{\mathrm{a}}$ à $5^{\mathrm{a}}$ falange distal.

Tabela 4. Soma da quantidade de linhas e tipo de desenho, por dedo, em indivíduos do grupo controle $(n=31)$.

\begin{tabular}{|c|c|c|c|c|c|c|c|c|}
\hline & \multirow[t]{2}{*}{ Média } & \multirow[t]{2}{*}{ S.E.M. } & \multirow{2}{*}{$\begin{array}{l}\text { Desvio } \\
\text { Padrão }\end{array}$} & \multirow[t]{2}{*}{ Amplitude } & \multirow[t]{2}{*}{ Variância } & \multicolumn{3}{|c|}{ Predominância (\%) } \\
\hline & & & & & & L & W & $A$ \\
\hline Mesql1 & 14,42 & 1,20 & 6,67 & 26 & 33,48 & 64,5 & 25,8 & 9,7 \\
\hline Mesql2 & 10,55 & 1,02 & 5,66 & 18 & 26,73 & 54,8 & 38,7 & 6,5 \\
\hline Mesql3 & 12,13 & 1,15 & 6,41 & 21 & 45,63 & 71,0 & 19,3 & 9,7 \\
\hline Mesql4 & 13,74 & 0,97 & 5,38 & 19 & 28,26 & 71,0 & 25,8 & 3,2 \\
\hline Mesql5 & 13,13 & 0,83 & 4.60 & 17 & 17,25 & 93,6 & 3,2 & 3,2 \\
\hline Mdsql1 & 16,19 & 1,07 & 5,96 & 26 & 29,17 & 51,6 & 41,9 & 6,5 \\
\hline Mdsql2 & 10,68 & 1,15 & 6,38 & 21 & 28,20 & 64,5 & 22,6 & 12,9 \\
\hline Mdsql3 & 11,16 & 1,00 & 5,59 & 20 & 31,81 & 87,0 & 6,5 & 6,5 \\
\hline Mdsql4 & 14,91 & 1,03 & 5,75 & 19 & 32,50 & 67,7 & 29,1 & 3,2 \\
\hline Mdsql5 & 13,71 & 0,86 & 4,80 & 15 & 20,13 & 90,3 & 6,5 & 3,2 \\
\hline
\end{tabular}


Tabela 5. Soma da quantidade total de linhas e intensidade de deltas em portadores da SAOS e no grupo controle.

\begin{tabular}{lcccccc}
\hline & & Média & S.E.M. & Desvio Padrão & Amplitude & Variância \\
\hline Apnéicos & SQTL & 129,03 & 6,14 & 34,21 & 131 & 1170,03 \\
$(\mathrm{n}=31)$ & D10 & 12,39 & 0,58 & 3,22 & 14 & 10,38 \\
Controles & SQTL & 130,03 & 7,50 & 41,78 & 151 & 1387,99 \\
$(\mathrm{n}=31)$ & D10 & 11,55 & 0,58 & 3,22 & 10 & 7,00 \\
\hline
\end{tabular}

SQTL, Somatória da quantidade de linhas nos dez dedos das mãos; D10, Índice de deltas para os dez dedos das mãos.

Compondo o grupo controle tivemos também 31 indivíduos saudáveis, isto é, sem sinais ou sintomas sugestivos de SAOS. A média de idade no grupo de apnéicos foi 45,9 ( $\pm 2,03)$ anos, coincidindo com o pico de incidência da síndrome situado entre 40 e 50 anos $^{21}$. Na Tabela 1 observa-se a estatística descritiva para o indice de apnéia e as características antropométricas do grupo estudado.

Não houve diferença estatisticamente significativa entre o valor médio da SQL para cada dedo dos apnéicos comparado ao seu correspondente no grupo controle. O mesmo ocorreu para os parâmetros SQTL e D10, quando comparados os valores médios encontrados nos dois grupos. Porém, numa análise da densidade de deltas por dedo, os dois grupos diferem na incidência dos mesmos, apesar desta diferença não ser significativa estatisticamente (Tabelas 2, 3 e 4).

$\mathrm{Na}$ Tabela 5 encontra-se a estatística descritiva para os parâmetros SQTL e D10 dos apnéicos e do grupo controle. A fórmula digital predominante para ambos os grupos foi LW, a qual incide em $45,2 \%$ dos apnéicos e em $35,5 \%$ dos controles. Para a fórmula ALW foi observada incidência em 9,7\% dos apnéicos e no grupo controle em $22,6 \%$. Observouse entre os apnéicos um dos indivíduos $(3,2 \%)$ com a fórmula $10 \mathrm{~W}$, porém, entre os controles nenhum deles a apresentou. Nenhuma destas diferenças entre os dois grupos mostrou-se estatisticamente significativa. Em relação à incidência dos tipos de desenho percebe-se na amostra estudada que os apnéicos apresentam mais verticilos do que os controles, porém, também de forma não significante estatisticamente. No Gráfico 1, visualiza-se a diferença entre os grupos na incidência dos tipos de desenho.

\section{DISCUSSÃO}

O valor médio encontrado para o IMC é considerado elevado $\left(>30 \mathrm{~kg} / \mathrm{m}^{2}\right)$, classificando os indivíduos da amostra como obesos. É sabido que o aumento

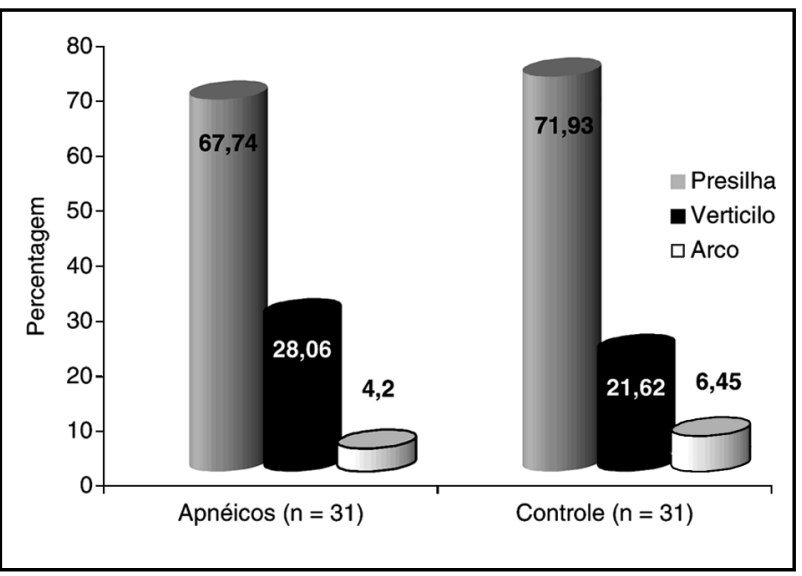

Gráfico 1. Percentual de incidência dos tipos de desenho nas impressão digitais.

do IMC pode ser um preditor para a SAOS ${ }^{22}$. Sendo a maior incidência da SAOS em adultos de meiaidade ${ }^{20}$, o único parâmetro do IMC a se alterar nestes indivíduos é a massa corporal, pois eles já têm sua estatura consolidada. Uma limitação do IMC é a de exprimir a relação entre massa corporal e estatura sem analisar a composição desta massa. Por isso, faz-se necessário observar tal composição nos portadores da SAOS para que haja clareza sobre a contribuição de cada componente no aumento do IMC.

A gordura corporal relativa do grupo amostral teve valor médio elevado, classificando-os também como obesos, porém, em obesidade de grau "leve" para as mulheres e de grau "elevado" para homens ${ }^{23}$. Sendo a amostra composta por maioria de homens $(71 \%)$, justifica-se a tendência da gordura corporal se distribuir mais em torno da região tóraco-abdominal. Um indicativo deste tipo de distribuição tipicamente central para a gordura, é a relação cintura-quadril (RCQ), já utilizada anteriormente $^{24}$. Pesquisadores observaram 1464 homens com SAOS e constataram que $28 \%$ deles eram obesos (IMC > 30) e 47\% tinham sobrepeso (IMC: 26-30), com índice médio para a RCQ de 0,99, apresentando 
cerca de $80 \%$ da amostra apresentou valores maiores que 0,94 . No presente estudo foi observado na amostra um valor médio para a RCQ de 0,92.

Outro parâmetro indicativo de obesidade central com similar valor clínico ao da RCQ é o indice de conicidade (IC), que entre os indivíduos da amostra apresentou valor médio considerado elevado ${ }^{18}$. Uma vantagem do IC é que o perímetro da cintura está corrigido pela massa corporal e a estatura, enquanto que a RCQ não associa os dois últimos parâmetros à distribuição da gordura corporal. Por ser a obesidade apenas um dos componentes da complexa e multifatorial etiologia da SAOS, os procedimentos terapêuticos existentes variam de redução do peso corporal a intervenções cirúrgicas como traqueostomia, uvulopalatofaringoplastia ${ }^{25}$ e gastroplastia ${ }^{26}$. Esta última é utilizada como alternativa para redução do peso em obesos com SAOS. São usados também de aparelhos dentários ${ }^{27}$ e máscara nasal com pressão positiva contínua (NCPAP) ${ }^{28}$.

Através do perfil somatotípico observa-se qual a predominância das massas óssea, muscular e adiposa na composição corporal dos apnéicos. Para o grupo estudado, a predominância da categoria endomorfo-mesomorfo indica elevada adiposidade relativa, gordura subcutânea abundante e maior acúmulo de gordura no abdômen; alto desenvolvimento músculo-esquelético relativo, com grandes diâmetros ósteo-articulares e grande volume muscular, e também, linearidade relativa de grande volume por unidade de altura, com o tronco e extremidades arredondadas, sendo estas últimas relativamente volumosas em relação ao primeiro.

Ao se utilizar os testes de regressão linear percebe-se que, dentre as variáveis antropométricas estudadas, o IMC foi considerada a melhor variável explanatória e a única aceita estatisticamente $(r=$ $0,612 ; p<0,05)$ como possível preditora para a SAOS. O valor de correlação do percentual de gordura corporal com o índice de apnéia (IA/h) foi estatisticamente não significante $(r=0,253 ; p<0,05)$. Considerando o tamanho da amostra e apesar de nenhuma outra variável antropométrica ter sido aceita estatisticamente como preditora, o indice de conicidade (IC) atingiu valor de correlação próximo ao do IMC, porém estatisticamente não significativo $(r=0,598 ; p<0,05)$ quando analisado simultaneamente com as outras variáveis. Se analisadas como preditoras isoladamente, percebe-se que dentre as variáveis o IMC tem a maior correlação $(r=0,649$; $\mathrm{p}<0,05)$, porém, o IC e o componente somatotípico mesomorfia mostram correlação estatisticamente significativa, sendo os valores de $(r)$ 0,560 e 0,552 $(p<0,01)$, respectivamente.

Numa análise mais detalhada, ao estratificarmos a amostra de apnéicos (casos) por nível de gravidade, observamos mudanças significativas na composição corporal dos indivíduos à medida que varia o nível de gravidade da síndrome. A Tabela 2 ilustra tais modificações.

As características dermatoglíficas dos apnéicos e do grupo controle são apresentadas e analisadas em duas etapas. Inicialmente, observa-se o valor médio para o número de linhas (SQL) e a predominância relativa de cada tipo de desenho $(A=a r c o ;$ $\mathrm{L}=$ presilha; $\mathrm{W}=$ verticilo) para cada dedo das mãos. Em seguida, observa-se a soma da quantidade total de linhas (SQTL); a intensidade de deltas (D10); a predominância da fórmula digital, que expressa a combinação entre os tipos de desenho encontrados; e a predominância geral para cada tipo de desenho (Arco, Presilha e Verticilo) entre os dois grupos. Nas Tabelas 3 e 4, observa-se a estatística descritiva da SQL e predominância do tipo de desenho, por dedo, para os apnéicos e o grupo controle, respectivamente.

Em conclusão, com base nos dados encontrados neste estudo e respeitando-se as limitações impostas pelo método, assim como as restrições observadas em função do tamanho amostral, chega-se às conclusões parciais seguintes. Os resultados sugerem que o aumento da gordura corporal relativa em indivíduos ditos "pesados", ou seja, com elevada densidade corporal, possa favorecer o surgimento de sintomas sugestivos da SAOS. Entre os apnéicos (casos) o estado de obesidade e/ou excesso de peso parece existir não só em razão do aumento da gordura, principalmente na região abdominal, mas também por sua constituição osteo-muscular mais densa. A utilização do indice de conicidade na análise da composição corporal parece ser interessante, dada sua promissora correlação com o índice de apnéia. Certamente em uma amostra maior podese avaliar melhor não só o valor preditivo desta variável antropométrica, mas também inferir de forma mais clara e consistente sobre a predominância dos tecidos adiposo, muscular e ósseo na constituição da massa corporal total. É recomendável que na continuidade do estudo seja observada a composição corporal de acordo com o nível de gravidade. Assim será possível verificar se há diferença na composição corporal dos grupos estratificados à me- 


\section{dida que varia o nível de gravidade. Através do teste t de Student, não foi encontrada diferença estatis- ticamente significativa na comparação entre as ca- racterísticas dermatoglíficas quantitativas e qua- litativas dos apnéicos e do grupo controle.}

\section{REFERÊNCIAS}

1. Victor LD. Obstructive sleep apnea. Am Family Physician 1999;60:2279-86.

2. Reimão R, Lemmi H. Narcolepsia e apnéia do sono concomitantes registro de caso. Arq Neuropsiquiatr 1986;44:73-77.

3. Reimão R, Lemmi H, Bertorini T. Sonolência excessiva diurna, apneia do sono tipo central e distrofia miotonica. Arq Neuropsiquiatr 1985; 43:391-395.

4. Reimão R, Papaiz GE, Papaiz LF. Pierre Robin sequence and obstructive sleep apnea . Arq Neuropsiquiatr 1994;52:554-559.

5. Redline S, Tosteson T, Tishler PV, Carskadon MA, Millman RP. Studies in the genetics of obstructive sleep apnea: familial aggregation of symptoms associated with sleep-related breathing disturbances. Am Rev Resp Dis 1992;145:440-444.

6. Ziegler AG, Mathies R, Ziegelmayer G, et al. Dermatoglyphics in type 1 diabetes mellitus. Diabet Med 1993;10:720-724.

7. Beiguelman B. Citogenética humana. Rio de Janeiro: Guanabara Koogan, 1982.

8. Lilienfeld DE, Stolley PD, Lilienfeld AM. Foundations of epidemiology. 3.Ed. New York: Oxford Univ Press, 1994;151-251.

9. Fukugawa NK, Bandini LG, Young JB. Effect of age on body composition and resting metabolic rate. Am J Physiol 1990;259:E233-E238.

10. Pollock ML, Wilmore JH. Exercícios na saúde e na doença. 2.Ed. Rio de Janeiro: Medsi 1993.

11. Lohman TG, Boileau RA, Massey BH. Prediction of lean body weight in young boys from skinfold thickness and body weight. Human Biol $1975 ; 47: 245-262$

12. Jackson AS, Pollock ML. Generalized equations for predicting body density of men. Brit J Nutr 1978;40:497-504
13. Jackson AS, Pollock ML, Ward A. Generalized equations for predicting body density of women. Med Sci Sports Exerc 1980;12:175-182.

14. Carter JEL, Heath BH. Somatotyping-development and applications. Cambridge: Cambridge Univ Press, 1990.

15. Mazza JC. Antropometrica. Norton K, Olds T (eds). Rosario: Biosystem Servicio Educativo, 2000.

16. Valdez R. A simple model-based index of abdominal adiposity. J Clin Epidemiol 1991;44:955-956.

17. Fernandes J Filho. A prática da avaliação física. Rio de Janeiro: Shape, 1999.

18. Valdez R, Seidell JC, Ahn YI, Weiss KM. A new index of abdominal adiposity as an indicator of risk for cardiovascular disease: a crosspopulation study. Int J Obesity 1992;17:77-82.

19. Cummins H, Midlo C. Finger prints, palms and soles An introduction to dermatoglyphics. Philadelphia: Blakiston,1943.

20. Reimão R, Joo SH. Apnéia obstrutiva do sono: diagnóstico e escolha terpêutica. Diag Trat. 1997;2:35-37.

21. Wiegand L, Zwilich CW. Obstructive sleep apnea. In Bone RC (ed.) Disease-a-Month. St. Louis: Mosby Year Book, 1994;40:199-252.

22. Blazejova K, Sonka K, Skodova Z, Nevsimalova S. Prevalence of obesity, hypertension and smoking in patients with the sleep apnea syndrome: comparison with the Czech population. Cas Lesk Cesk 2000;139:339-342.

23. Guedes DP, Guedes JERP. Controle do peso corporal: composição corporal atividade física e nutrição. Londrina: Midiograf, 1998.

24. Grunstein R, Wilcox I, Yang TS, Gould Y, Hedner J. Snoring and sleep apnea in men: association with central obesity and hypertension. Int J Obes Rel Met Dis 1993;17:533-540.

25. Reimão R, Lemmi H, Ariskal H, Cocke E. Acompanhamento polissonográfico das apneias do sono apos uvulopalatofaringoplastia. Arq Neuropsiquiatr 1985,43:360-364

26. Reimão R, Lemmi H, Cowan G, Golden EB. Papel da gastroplastia no tratamento da apneia. Narcolepsia e apnéia do sono obstrutiva durante o sono. Arq Neuropsiquiatr 1986,44:38-43.

27. Reimão R, Gouveia MM, Pestana MCG, et al. Obstructive sleep apnea treatment with dental appliance. Arq Neuropsiquiatr 1994,52:560-565.

28. Silva AB, Lemmi H. NCPAP for the treatment of obstructive sleep apnea. Arq Neuropsiquiatr 1989,47:150-152. 